\title{
Prevalence of COPD in India: a systematic review
}

\author{
*Ailsa J McKay", P A Mahesh', Julia Z Fordhama, Azeem Majeed \\ a Imperial College School of Medicine, Imperial College London, London, UK

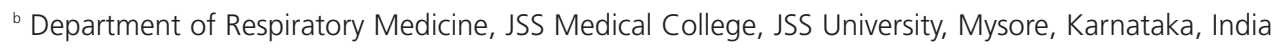 \\ c Department of Public Health and Primary Care, Imperial College London, London, UK
}

Received 28th February 2012; revised 26th April 2012; accepted 11th June 2012; online 13th July 2012

\begin{abstract}
Background: The increasing burden of chronic diseases is a particular risk to countries with developing health systems. Chronic obstructive pulmonary disease (COPD) is contributing to the burden of chronic diseases. Understanding the current prevalence of COPD in India is important for the production of sustainable management strategies.

Aims: To provide a systematic review of studies assessing the prevalence of COPD in India.

Methods: Database searches, journal hand searches, and scanning of reference lists were used to identify studies. Studies of general adult populations resident in India were included. Data extraction and quality assessment were carried out using pre-tested proformas. Owing to the heterogeneity of reviewed studies, meta-analysis was not appropriate. Thus, narrative methods were used.

Results: We did not identify any studies from which we could draw a rigorous estimate of the prevalence of COPD by standard definition. Reliable standard estimates of chronic bronchitis were only available for rural populations. We identified four studies that gave estimated prevalences between $6.5 \%$ and $7.7 \%$, and others suggestive that prevalences in some environmentally atypical regions may lie outside this range. Sex and smoking status were relatively important predictors of COPD prevalence. Residential environs, age, and domestic smoke exposure are also important, but investigation of their effect was limited by study heterogeneity.

Conclusions: Although limited by the number and heterogeneity of studies and their unsuitability for meta-analysis, we found the most rigorous existing estimates of the general prevalence of chronic bronchitis in rural areas to lie between $6.5 \%$ and $7.7 \%$. These figures are unlikely to apply to all Indian subpopulations, so the general prevalence of chronic bronchitis in India remains unknown. Accurate estimates of the prevalence of chronic bronchitis/COPD from across the country are required to supplement existing data if optimal management strategies are to be devised.
\end{abstract}

(C) 2012 Primary Care Respiratory Society UK. All rights reserved.

AJ McKay et al. Prim Care Respir J 2012; 21(3): 313-321

http://dx.doi.org/10.4104/pcrj.2012.00055

Keywords COPD, chronic disease prevalence, India, systematic review

See linked editorial by Salvi et al. on pg 249

The full version of this paper, with online appendix,

is available online at www.thepcrj.org

\section{Introduction}

Chronic obstructive pulmonary disease (COPD) is one of several chronic diseases that are becoming increasingly problematic worldwide. Their increasing burden and monetary cost are a particular risk to low- and middle-income countries. If trends continue unabated, chronic diseases have the potential to overwhelm health systems and state economies. Several high-level international organisations have expressed their concern about the impact of chronic diseases on health systems. ${ }^{1-3}$

Low- and middle-income countries already shoulder much of the burden of COPD. Ninety percent of COPD deaths occur in these countries ${ }^{4}$ - not insignificant when COPD accounted for $5 \%$ of deaths (globally) in $2005^{4}$ - and COPD is predicted to become the third leading cause of death worldwide by $2030 .{ }^{5}$ These countries also suffer higher numbers of COPD-associated disability-adjusted life years. ${ }^{6}$

The reasons why chronic diseases are becoming so problematic are certainly associated with increasing prevalences in some cases, but this has not been well established for COPD.? The impact of COPD on health systems could also be increasing due to a greater ability and capacity to manage the disease, the frequency of exacerbations, and enhanced survival of patients.

\footnotetext{
* Corresponding author: Dr Ailsa McKay, Imperial College School of Medicine, Imperial College London, London SW7 2AZ, UK. Tel: +44 (0)20 85395522 E-mail: ailsa.mckay08@imperial.ac.uk
} 


\section{COPD in India}

Each of these considerations may be relevant in India where little is known regarding prevalence trends. Indeed, even the current prevalence is not well understood, although a series of studies collected by Murthy and Sastry ${ }^{8}$ and Jindal ${ }^{9}$ have suggested that it may average around $5 \%$ in the adult population with higher rates in smokers, males, rural areas, depending on the type of domestic fuel use and socioeconomic status. The size and diversity of the Indian population hinders prevalence estimates, but they are important as COPD inflicts a relatively high morbidity on India. ${ }^{6}$ Satisfactory prevalence estimates and reasonable future predictions will help India to manage this problem adequately.

\section{Review aims}

We aimed to review the prevalence of COPD in India. This has not, to our knowledge, been the subject of a systematic review previously, and scoping searches indicate that several study results have become available since the most recent published reviews of the topic in 2005 and 2006.,9

We aimed to address the following questions:

1. What is the prevalence of COPD in India?

2. What has been the trend in prevalence across the last 30 years?

3. Are prevalence/time trends associated with age, sex, urban/rural location, smoking status and/or domestic fuel exposure?

\section{Methods}

The review was conducted according to the relevant aspects of the PRISMA guidance ${ }^{10}$ (see Appendix available online at www.thepcrj.org). Following scoping searches a review protocol was developed, describing the search strategy and methods for data collection and analysis.

The review questions above were deconstructed to identify PICOS elements used to generate search terms (see Table 1). The search strategy was trialled before use to determine that existing related well-cited articles would be returned and changes made before the final search was performed.

\section{Search}

The PICOS identifiers in the research questions and related terms listed in Table 1 were used to search the Medline and Embase databases via PubMed and Ovid, respectively. The search was carried out on 8 July 2011 and updated on 22 December 2011 without restriction on language of publication, study design, publication type, or publication status. Only studies published since 1980 were included as our interest was in the recent situation, and earlier publications were also relatively few and difficult to access. In addition, the available online content (from 1980 onwards) of the journals Respiratory Medicine: COPD Update, COPD: Journal of COPD, International Journal of COPD, Lung India, Indian Journal of Chest Diseases and Allied Sciences, Respiratory Medicine, Thorax, and the National Medical Journal of India were hand searched during June 2011. Reference lists of included papers, as well as review articles/editorials identified in the searches, were scanned.

\section{Selection}

We included studies of general populations from any area of India. Studies that estimated the prevalence of chronic bronchitis were also included as this has been a commonly used proxy in epidemiological studies owing to its relative ease of diagnosis. Studies of specific occupational groups, hospital/clinic-attending populations, and review articles without novel synthesis were excluded.

To determine study eligibility, the titles and abstracts of all search results were screened by one reviewer. Studies accepted at this point were reviewed fully and progressed either to data extraction or exclusion. Exclusions were made according to the criteria above and where the available data were not COPD-specific (see Figure 1).

\section{Data extraction}

Two separate proformas were used to extract the basic study data and quality-related data (see Box 1). The quality proforma was a modified version of the checklist recommended by the Centre for Reviews and Dissemination, York. ${ }^{11}$ The proformas were tested on a subset of the papers and iterations made before being used for final data collection.

One reviewer carried out the extraction of basic study data. The results were checked by a second reviewer and concerns remedied by rechecking to reach a consensus decision. Two reviewers independently performed the extraction of quality data before the results were collated. Inconsistencies were resolved by discussion with an independent reviewer.

Prevalence data were recorded as overall general population prevalence where possible. Study of the effects of the major COPD risk factors (smoking and domestic smoke exposure) and important determinants (age, sex and location of residence) on prevalence were also noted.

Table 1. PICOS identifiers from research questions ('key terms') and database- and thesaurus- derived alternatives ('additional terms') used to generate database searches

\begin{tabular}{|c|c|c|c|c|c|c|}
\hline & $P$ & 1 & C & 0 & & $S$ \\
\hline Key terms & $\begin{array}{l}\text { All populations } \\
\text { resident in India }\end{array}$ & $\mathrm{n} / \mathrm{a}$ & $\mathrm{n} / \mathrm{a}$ & Prevalence & COPD & All \\
\hline
\end{tabular}

Asterisks indicate where all database terms based on the attached stem were included. Terms within each column were distinguished using the OR function, and the terms in different columns combined using the AND function. 
Figure 1. Flow chart demonstrating handling of papers returned by Search 1. Chart adapted from Moher et al. ${ }^{10}$

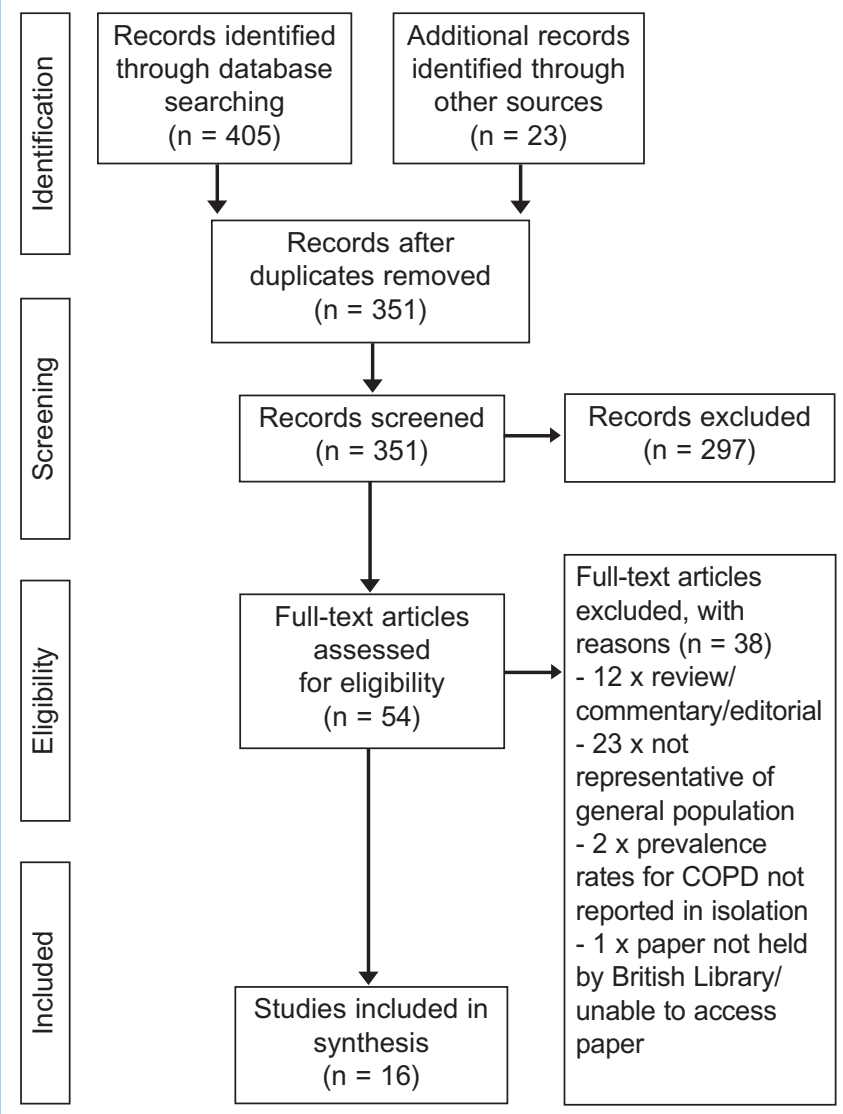

\section{Quality assessment}

Two reviewers used the extracted quality data to independently grade each aspect of the assessment as 'good', 'poor', or 'nonassessable'. Disagreements were resolved by discussion and consensus decision. Where the combination of the number of 'poor'/'non-assessable' ratings and main reasons for concern were deemed sufficient to question the reliability of the study, the study was excluded from a secondary analysis (see below).

\section{Data synthesis}

As anticipated on preliminary searching, study designs were too heterogeneous to allow useful meta-analysis. Thus, a narrative approach was used. The data were summarised in tables and analysed twice (before and after exclusion of studies where quality was of concern).

We aimed to assess the studies with regard to:

- Number of studies and time trends in production and quality

- Time trends in prevalence

- Prevalence by subgroup (age, sex, urban/rural location, smoking status, domestic fuel exposure)

Proposals were formed based on general initial analysis outcomes and inconsistencies examined. Finally, comparisons were made between results of the analyses before and after studies with quality
Box 1. Data extraction and quality assessment checklists. The numbers beside the quality assessment criteria are used to indicate the 11 points used to generate the quality scores presented in Tables 2 and 3

\begin{tabular}{|c|c|}
\hline General data extraction & Quality assessment checklist \\
\hline $\begin{array}{l}\text { - Study dates (or publication } \\
\text { date if not available) } \\
\text { - Study design } \\
\text { - Type of report } \\
\text { - Number of participants } \\
\text { (enrolled, excluded and lost } \\
\text { to follow up) } \\
\text { - Participant characteristics } \\
\text { (including age, sex, smoking } \\
\text { status, exposure to domestic } \\
\text { fuels, and socioeconomic } \\
\text { status, where available) } \\
\text { - Study setting (location, and } \\
\text { urban or rural) } \\
\text { - Definition of diagnosis used } \\
\text { - Measurement/assessment } \\
\text { tool } \\
\text { - Outcomes (including } \\
\text { subgroup data for age, sex, } \\
\text { urban/rural residence, } \\
\text { smoking status and domestic } \\
\text { fuel exposure where } \\
\text { available) }\end{array}$ & $\begin{array}{l}\text { 1. Type of report } \\
\text { 2. Clear aims/objectives } \\
\text { 3. Clear and appropriate } \\
\text { methods, including } \\
\text { sampling/recruitment (4), } \\
\text { inclusion/exclusion criteria } \\
\text { (5), and data collection } \\
\text { 6. Appropriate and rigorous } \\
\text { analysis } \\
\text { 7. Outcomes not reported, or } \\
\text { additional outcomes } \\
\text { reported } \\
\text { 8. Risk of bias in selection } \\
\text { 9. Risk of bias in } \\
\text { measurement and } \\
\text { outcomes } \\
\text { 10. Limitations discussed } \\
\text { 11. Funding information and } \\
\text { information regarding } \\
\text { conflicts of interest }\end{array}$ \\
\hline
\end{tabular}

concerns were excluded. Where data were insufficient to allow narrative synthesis, display and discussion of the data sufficed.

\section{Results}

Our initial search identified 351 papers for review. Figure 1 displays the handling of search results; 16 papers were finally deemed suitable for inclusion. The data extracted from studies without quality concerns are summarised in Table 2, and the remainder in Table 3, with the main quality concerns noted. Of the 16 papers reviewed, five were undertaken/published in the 1980s, four in the 1990s, and seven in the 2000s. Quality ratings were generally higher in more recent studies.

The possibility of a time trend in study quality and further variability between studies made it difficult to consider time trends in COPD prevalence. The studies reviewed were undertaken in urban, rural and mixed populations, in sites reaching from Kashmir to Tamil Nadu. The age ranges of the studied populations were varied, and the male portion of the populations ranged from $39 \%$ to $68 \%$ - especially important given the differential prevalence of smoking between men and women (see Tables 2 and 3). Where reported, rates of 'current smoking' varied between 3.8\% and $73.1 \%$ in males and $0 \%$ and $28 \%$ in females, and rates of domestic smoke exposure between $36 \%$ and 'ubiquitous'.

The definition of COPD was a further complication in the comparison of prevalence estimates. Traditionally, chronic bronchitis is defined as cough with expectoration 'occurring on most days for at least 3 months of the year for at least 2 consecutive years ${ }^{\prime 28}$ and 


\begin{tabular}{|c|c|c|c|c|c|}
\hline Ref & $\begin{array}{l}\text { Study dates/ } \\
\text { location }\end{array}$ & $\begin{array}{l}\text { Population size, characteristics } \\
\text { and smoke/pollution exposure }\end{array}$ & $\begin{array}{l}\text { i. Disease assessed (COPD or CB) } \\
\text { ii. Methods of assessment (if not } \\
\text { questionnaire) } \\
\text { iii. Definition of disease }\end{array}$ & Outcomes & Quality \\
\hline 12 & $\begin{array}{l}\text { P2010; } \\
\text { Karnataka (R) }\end{array}$ & $\begin{array}{l}\mathrm{n}=900 ; 50.3 \% \text { male; }>40 \text { years } \\
\text { Ever smokers: } 71.9 \% \text { males; } 0 \% \\
\text { females (but } 55 \% \text { passive); }>20 \\
\text { years biomass fuel exposure: } \\
80.9 \% \text { females }\end{array}$ & $\begin{array}{l}\text { i. } C B \\
\text { iii. }>40 \text { years, smoke/fuels exposure, and } \\
\text { whistling in chest/breathless/cough for } \geq 3 \\
\text { months/year for } \geq 2 \text { years }\end{array}$ & $\begin{array}{l}\text { Overall prevalence: } 7.1 \% \text { ( } 11.1 \% \text { males; } \\
4.5 \% \text { females) } \\
\text { † prevalence in smokers, males, and with } \\
\uparrow \text { age }(S) \text {; additive effects of domestic fuels } \\
\text { and passive smoking (S) }\end{array}$ & $\begin{array}{l}\text { G: } 10 \\
\text { P: } 1\end{array}$ \\
\hline 13 & $\begin{array}{l}\text { P2006; } \\
\text { various } \\
\text { locations } \\
U+R\end{array}$ & $\begin{array}{l}\mathrm{n}=35295 ; 51.6 \% \text { males; }>35 \\
\text { years; } 52 \% \text { U; smokers: } 43.8 \% \\
\text { males, } 4 \% \text { females; } 36 \% \\
\text { cooking fuel exposed }\end{array}$ & $\begin{array}{l}\text { i. } C B \\
\text { iii. productive cough on most of the days } \\
\text { for at least three months in a year for } \geq 2 \\
\text { consecutive years }\end{array}$ & $\begin{array}{l}\text { Overall: } 4.1 \% \text { ( } 5 \% \text { males; } 3.2 \% \text { females) } \\
\uparrow \text { prevalence in males, (passive) smokers, } \\
\text { with } \uparrow \text { age, lower SES, R residence (S); } \\
\text { additive effects of passive smoking and solid } \\
\text { fuel use (S) }\end{array}$ & $\begin{array}{l}\text { G: } 10 ; \\
\text { P: } 1\end{array}$ \\
\hline 14 & $\begin{array}{l}2003-5 \\
\text { unclear U + } \\
\text { R locations }\end{array}$ & $\begin{array}{l}n=\text { between } 2000-3000 ; \\
\text { between } 34 \text { and } 47 \% \text { male; }>65 \\
\text { years }\end{array}$ & $\begin{array}{l}\text { i. CB ( although unclear) } \\
\text { iii. morning productive cough for at least } \\
3 \text { months of year, plus 'clinical assessment' }\end{array}$ & $\begin{array}{l}\text { U prevalence: } 1.8 \% \\
\text { R prevalence: } 7.6 \%\end{array}$ & $\begin{array}{l}\text { G: 10; } \\
\text { N: } 1\end{array}$ \\
\hline 15 & $\begin{array}{l}\text { 2002-3; } \\
\text { Dibrugarh } \\
\text { (U) }\end{array}$ & $\begin{array}{l}\mathrm{n}=293 ;>60 \text { years; } 61.8 \% \text { male; } \\
\text { current smokers: } 3.8 \% \text { males, } \\
1.8 \% \text { females }\end{array}$ & $\begin{array}{l}\text { i. CB } \\
\text { iii. productive cough } \pm \text { wheeze for } \geq 3 \\
\text { months of } \geq 3 \text { consecutive years }\end{array}$ & $\begin{array}{l}\text { Overall prevalence: } 7.5 \% \text { ( } 8.8 \% \text { males; } \\
5.4 \% \text { females) } \\
\text { Smokers ' } 2.7 \times \text { more likely to have } \\
\text { respiratory disease' }\end{array}$ & $\begin{array}{l}\text { G: } 6 \text {; } \\
\text { P: } 1 ; \\
\text { N: } 4\end{array}$ \\
\hline 16 & $\begin{array}{l}\text { 2001-2; } \\
\text { U + R H. } \\
\text { Pradesh }\end{array}$ & $\begin{array}{l}n=1330(665 \text { each } U+R) \\
59.47 \% \text { male; }>18 \text { years }\end{array}$ & $\begin{array}{l}\text { i. CB } \\
\text { iii. 'defined based on clinical case definition } \\
\text { given by MRC' }\end{array}$ & $\begin{array}{l}\text { Overall prevalence: } 9.1 \% \text { ( } 11.1 \% \text { males; } \\
6.1 \% \text { females) } \\
\uparrow \text { prevalence in males, smokers, with } \uparrow a g e, \\
R \text { residence, use of wood/cow dung fuels }(\mathrm{S})\end{array}$ & $\begin{array}{l}\text { G: } 5 \\
\text { P: } 2 \\
\text { N: } 4\end{array}$ \\
\hline 17 & $\begin{array}{l}\text { P2001; } \\
\text { Delhi (U) }\end{array}$ & $\begin{array}{l}n=4171 ;>18 \text { years; } 56.6 \% \\
\text { male } \\
\text { All lived in same area for at least } \\
10 \text { years }\end{array}$ & $\begin{array}{l}\text { i. CB (NB. no separate data for COPD } \\
\text { alone) } \\
\text { ii. Questionnaire and spirometry } \\
\text { iii. productive cough most days for } \geq 3 \\
\text { consecutive months over past } \geq 2 \text { years }\end{array}$ & $\begin{array}{l}\text { Prevalence (lower-pollution zone): } \\
0.8-3.1 \% \text { in males, } 0.3-2.1 \% \text { in females } \\
\text { Prevalence (higher-pollution zone): } \\
0.5-4.6 \% \text { in males; } 1.1-5.9 \% \text { in females } \\
\text { No prevalence difference between pollution } \\
\text { zones }\end{array}$ & $\begin{array}{l}\text { G: } 7 \\
P: 4\end{array}$ \\
\hline 18 & $\begin{array}{l}\text { 1999-2000; } \\
U+R \\
\text { Haryana } \\
\text { State }\end{array}$ & $\begin{array}{l}n=200(100 \text { each } U+R) ;>60 \\
\text { years; } 49 \% \text { male }(U+R)\end{array}$ & $\begin{array}{l}\text { i. COPD } \\
\text { ii. History, examination, medical records, } \\
\text { medications } \\
\text { iii. Based on reported illness, examination, } \\
\text { records, medication }\end{array}$ & $\begin{array}{l}\text { Overall prevalence: } 42 \% \\
\text { U population: } 36.7 \% \text { males, } 31.4 \% \text { females } \\
\text { R population: } 57.1 \% \text { males, } 43.1 \% \text { females }\end{array}$ & $\begin{array}{l}\text { G: } 10 ; \\
\mathrm{N}: 1\end{array}$ \\
\hline 19 & $\begin{array}{l}\text { P1994; } \\
\text { Kashmir (R) }\end{array}$ & $\begin{array}{l}\mathrm{n}=286 \text { Gujjars, } 274 \text { non-Gujjars; } \\
\text { >15 years; region 'free } \\
\text { from... pollution' } \\
\text { Gujjars: } 54.2 \% \text { male; } 58.04 \% \\
\text { well ventilated homes; } 21.33 \% \\
\text { smokers (all male); non-Gujjars: } \\
49.6 \% \text { male; } 95.99 \% \text { well } \\
\text { ventilated homes; } 41.61 \% \\
\text { smokers (most male) }\end{array}$ & $\begin{array}{l}\text { i. CB } \\
\text { ii. History, plus spirometry if } C B \text { diagnosis } \\
\text { iii. CB: 'productive cough on most days } \geq 3 \\
\text { consecutive months of } 2 \text { successive years' } \\
\text { (NB. spirometry performed, but no } \\
\text { definitions of airway obstruction given) }\end{array}$ & $\begin{array}{l}\text { Overall prevalence CB: } 7.68 \% \text { (Gujjars: } \\
10.14 \% \text {, non-Gujjars: } 5.11 \% \text { (difference S, } \\
\text { in both sexes)) } \\
\uparrow \text { prevalence in: low SES, overcrowding, } \\
\text { Gujjar smokers (NS), non-Gujjar smokers, } \\
\text { lower housing class, poorly ventilated } \\
\text { homes, and } \uparrow \text { domestic smoke exposure (S) }\end{array}$ & $\begin{array}{l}\text { G: } 5 \\
\text { P: } 3 \\
\text { N: } 3\end{array}$ \\
\hline 20 & $\begin{array}{l}\text { P1982; } \\
\text { Uttar } \\
\text { Pradesh (R) }\end{array}$ & $\begin{array}{l}\mathrm{n}=1424 ;>20 \text { years; } \\
54.4 \% \text { male; current smokers: } \\
73.1 \% \text { males, } 12.0 \% \text { females; } \\
\text { never smokers: } 14.8 \% \text { males, } \\
85.8 \% \text { females }\end{array}$ & $\begin{array}{l}\text { i. CB } \\
\text { iii. 'history of productive cough for about } \\
3 \text { months a year, during last } 2 \text { years' }\end{array}$ & $\begin{array}{l}\text { Overall prevalence CB: } 6.5 \% \text { ( } 8.1 \% \text { males, } \\
4.5 \% \text { females) } \\
\text { Higher prevalence in males (S) and } \\
\text { smokers (S) }\end{array}$ & $\begin{array}{l}\text { G: } 7 \\
\text { P: } 2 \\
\text { N: } 2\end{array}$ \\
\hline
\end{tabular}

U=urban; R=rural; MRC=Medical Research Council; SES=socioeconomic status; NS=non-significant; S=significant; $G=$ good; $P=$ poor; $N=$ non-assessable'

COPD is defined as this plus spirometric evidence of chronic airway obstruction. ${ }^{29}$ Five studies reported so-defined prevalences of chronic bronchitis rather than COPD. . $3,16,17,19,20$ No studies reported prevalences of COPD alone, but five reported those of chronic bronchitis and COPD. ${ }^{22,23,25-27}$ The remaining six studies reported prevalences of COPD and/or chronic bronchitis with less traditional ${ }^{12,15,18}$ or illdefined ${ }^{14,15,21,24}$ definitions. Table 4 lists prevalence estimates of COPD and chronic bronchitis separately and identifies the estimate as deriving from an urban and/or rural population.

\section{Prevalence of COPD}

The results obtained were varied. This is likely to be at least partly due to definitions of disease. For example, one study found the prevalence of airway obstruction to be relatively high (58.5\%), but it is not clear that all obstruction was associated with symptoms so it 


\begin{tabular}{|c|c|c|c|c|c|}
\hline Ref & $\begin{array}{l}\text { Study dates/ } \\
\text { location }\end{array}$ & $\begin{array}{l}\text { Population size, characteristics } \\
\text { and smoke/pollution exposure }\end{array}$ & $\begin{array}{l}\text { i. Disease assessed (COPD or CB) } \\
\text { ii. Methods of assessment } \\
\text { iii. Definition of disease }\end{array}$ & Outcomes & $\begin{array}{l}\text { Quality scores } \\
\text { (main concerns } \\
\text { in parentheses) }\end{array}$ \\
\hline 21 & $\begin{array}{l}\text { P2010; } \\
\text { West Bengal } \\
\text { (R) }\end{array}$ & $\begin{array}{l}\mathrm{n}=201 ; 39 \% \text { male; }>35 \text { years; } \\
\text { 'ubiquitous' indoor stove use }\end{array}$ & $\begin{array}{l}\text { i. COPD } \\
\text { ii. Questionnaire and spirometry } \\
\text { iii. Disease severity classified 'according to } \\
\text { British guidelines' }\end{array}$ & $\begin{array}{l}\mathrm{AO} \text { in } 58.5 \% \text { (' } 51 \% \text { mild, } 6 \% \\
\text { moderate, } 1.5 \% \text { severe) } \\
\text { 'history of current smoking...associated } \\
\text { with ... } \mathrm{AO}^{\prime}\end{array}$ & $\begin{array}{l}\text { G: } 1 ; \text { P: } 3 ; \text { N: } 7 \\
\text { (abstract; lack } \\
\text { of information) }\end{array}$ \\
\hline 22 & $\begin{array}{l}\text { P1999; } \\
\text { Kashmir (U) }\end{array}$ & $\begin{array}{l}\mathrm{n}=1140 ;>30 \text { years; } 55.1 \% \\
\text { male; cold area 'requiring } \\
\text { relatively much heating' } \\
\text { (kerosene, gasoline and other } \\
\text { fuels); } 52.3 \% \text { smokers }\end{array}$ & $\begin{array}{l}\text { i. CB and COPD } \\
\text { ii. History, chest examination, spirometry } \\
\text { if CB diagosis } \\
\text { iii. CB: Productive cough for } \geq 3 \\
\text { months/year over last } 2 \text { years; COPD: CB } \\
+ \text { FEV } 1 / \text { FVC: } 65-74 \% \text { (mild), } 50-64 \% \\
\text { (moderate) or }<50 \% \text { (severe) }\end{array}$ & $\begin{array}{l}\text { Overall prevalence CB: } 5.7 \% \\
\text { Overall prevalence COPD: } 5.2 \% \\
\text { (45.7\% mild, } 34.29 \% \text { moderate, } \\
11.4 \% \text { severe) } \\
\uparrow \text { prevalence with } \uparrow \text { age, in smokers, } \\
\text { and those with poorly ventilated homes } \\
\text { (S) }\end{array}$ & $\begin{array}{l}\text { G: } 2 ; \mathrm{P}: 4 ; \mathrm{N}: 5 \\
\text { (lack of } \\
\text { information } \\
\text { regarding } \\
\text { methods and } \\
\text { analysis) }\end{array}$ \\
\hline 23 & $\begin{array}{l}\text { 1990; } \\
\text { Chandigarh } \\
\text { City and } \\
\text { Mullanpur } \\
(U+R)\end{array}$ & $\begin{array}{l}\mathrm{n}=1475(846 \mathrm{U}, 629 \mathrm{R}) \\
>15 \text { years; } 39.3 \% \text { male } \\
\text { Males: } 24.9 \% \text { smokers }\end{array}$ & $\begin{array}{l}\text { i. CB and COPD } \\
\text { ii. Questionnaire, examination and PEFR } \\
\text { iii. CB: productive cough for } \geq 3 \\
\text { months/year for } \geq 2 \text { consecutive years; } \\
\text { COPD: CB plus PEFR < population mean } \\
\text { minus } 1.65 \text { SD }\end{array}$ & $\begin{array}{l}\text { Overall prevalence CB: } 3.6 \%(5 \% \\
\text { males, } 2.7 \% \text { females) } \\
\text { Prevalence higher in smokers and } \mathrm{R} \\
\text { women (S) } \\
\text { Overall prevalence COPD: } 1.8 \% \\
\uparrow \text { prevalence in smokers (S) }\end{array}$ & $\begin{array}{l}\text { G: 7; P: 2; N: } 2 \\
\text { (follow-up study, } \\
\text { population } \\
\text { characteristics } \\
\text { unclear; } \\
\text { selection } \\
\text { unclear) }\end{array}$ \\
\hline 24 & $\begin{array}{l}\text { P1987; } \\
\text { Mumbai } \\
(U+R)\end{array}$ & $\begin{array}{l}\mathrm{n}=4129 ; 50.3 \% \text { male: } \\
1 \text { to }>45 \text { years } \\
\text { High } \mathrm{SO}_{2} \text { area: } 21.4 \% \text { males } \\
\text { smokers, } 21 \% \text { wood/coal use; } \\
\text { medium } \mathrm{SO}_{2} \text { area: } 20.8 \% \text { males } \\
\text { smokers, } 6 \% \text { wood/coal use; low } \\
\mathrm{sO}_{2} \text { area: } 17.7 \% \text { males smokers, } \\
0.1 \% \text { wood/coal use; } \mathrm{R} \\
\text { population: } 15.6 \% \text { males } \\
\text { smokers, } 96 \% \text { wood/coal use }\end{array}$ & $\begin{array}{l}\text { i. 'CB' } \\
\text { ii. Questionnaire, examination and } \\
\text { spirometry } \\
\text { iii. Not provided, but questionnaire } \\
\text { 'patterned after BMRC respiratory } \\
\text { proforma 1976' }\end{array}$ & $\begin{array}{l}\text { Prevalence of CB: } 4.5,4.5,2.3 \text { and } \\
5.0 \% \text {, in high, medium and low } \mathrm{SO}_{2} \\
\text { and } \mathrm{R} \text { areas respectively } \\
\text { NB. Spirometry results for the COPD } \\
\text { population alone not provided }\end{array}$ & $\begin{array}{l}\text { G: } 1 ; \mathrm{P}: 7 ; \mathrm{N}: 3 \\
\text { (objectives, } \\
\text { analysis and } \\
\text { some elements } \\
\text { of methods } \\
\text { unclear) }\end{array}$ \\
\hline 25 & $\begin{array}{l}\text { 1981-86; } \\
\text { Tamil Nadu } \\
\text { (R) }\end{array}$ & $\begin{array}{l}\mathrm{n}=9946 ; \geq 30 \text { years; } 48.8 \% \\
\text { male; villages 'generally free } \\
\text { from atmospheric pollution'; no } \\
\text { smoking in females }\end{array}$ & $\begin{array}{l}\text { i. CB and COPD } \\
\text { ii. Questionnaire and PEFR } \\
\text { iii. CB: Productive cough } \pm \text { wheeze } \\
\text { lasting for } \geq 3 \text { months of } \geq 2 \text { consecutive } \\
\text { years; COPD: CB plus AO: } 60-69 \% \\
\text { (mild), } 50-59 \% \text { (moderate), or }<50 \% \\
\text { (severe)of normal }\end{array}$ & $\begin{array}{l}\text { Overall prevalence CB: } 3.3 \% ; \\
\uparrow \text { prevalence CB in males (4.1 vs 2.6\%) } \\
\text { and with } \uparrow \text { age (S) } \\
\text { Overall prevalence COPD: } 2.5 \% \text { (3.3\% } \\
\text { males, } 1.8 \% \text { females); mild: } 16.3 \% \\
\text { males, } 21.9 \% \text { females, moderate: } 17 \% \\
\text { males, } 18.1 \% \text { females, severe: } 47.7 \% \\
\text { males, } 31.4 \% \text { females; } \uparrow \text { prevalence } \\
\text { severe AO in smokers (S) }\end{array}$ & $\begin{array}{l}\text { G: } 4 ; \mathrm{P}: 2 ; \mathrm{N}: 5 \\
\text { (parts of } \\
\text { methods and } \\
\text { analysis unclear, } \\
\text { information from } \\
\text { family members } \\
\text { if participant } \\
\text { unavailable) }\end{array}$ \\
\hline 26 & $\begin{array}{l}\text { P1986; } \\
\text { Himachal } \\
\text { Pradesh (R) }\end{array}$ & $\begin{array}{l}\mathrm{n}=446 ; 68.2 \% \text { male; } 18-80 \\
\text { years; } 60.5 \% \text { males, } 28 \% \\
\text { females current smokers; poorly } \\
\text { ventilated homes; 'indoors most } \\
\text { of day for up to } 4-6 \\
\text { months/year' due to weather }\end{array}$ & $\begin{array}{l}\text { i. CB and COPD } \\
\text { ii. Questionnaire and spirometry } \\
\text { iii. CB: defined by MRC } \\
\text { questionnaire/criteria; COPD: CB plus } \\
\text { 'abnormal FEV } \text { 1' }^{\prime}(<1.5 \mathrm{~L} \text { for males, }<1 \mathrm{~L} \\
\text { for females) }\end{array}$ & $\begin{array}{l}\text { Overall prevalence CB: } 20.9 \% \text { ( } 21.7 \% \\
\text { males; } 19.0 \% \text { females); overall } \\
\text { prevalence COPD: } 8.3 \% \text { ( } 7.2 \% \text { males, } \\
10.5 \% \text { females) } \\
\uparrow \text { prevalence CB and COPD in males } \\
\text { (S), and with } \uparrow \text { age (significance } \\
\text { unclear) }\end{array}$ & $\begin{array}{l}\text { G: } 2 ; P: 4 ; N: 5 \\
\text { (lack of } \\
\text { information re. } \\
\text { methods and } \\
\text { analysis) }\end{array}$ \\
\hline 27 & $\begin{array}{l}\text { p1981; } \\
\text { Chandigarh } \\
\text { City (U) }\end{array}$ & $\begin{array}{l}\mathrm{n}=2825 ;>16 \text { years; } 51.3 \% \text { male; } \\
\text { non-smokers: } 69.4 \% \text { males; } \\
99.3 \% \text { females; smokers: } 30.6 \% \\
\text { males } \\
\text { Cooking habits of females: } \\
28.2 \% \text { fas; } 39.5 \% \text { kerosene; } \\
29.4 \% \text { kerosene + coal }\end{array}$ & $\begin{array}{l}\text { i. CB and COPD } \\
\text { ii. Questionnaire and PEFR } \\
\text { iii. CB: diagnosis by MRC } \\
\text { questionnaire/criteria; COPD: CB plus AO } \\
\text { (PEFR thresholds reported elsewhere, but } \\
\text { not available) }\end{array}$ & $\begin{array}{l}\text { Prevalence CB: non-smokers: } 0.89 \% \\
\text { males, } 1.6 \% \text { females; smokers: males: } \\
9.9 \% \\
\text { Prevlance COPD: } 0.60 \% \text { male non- } \\
\text { smokers; } 4.27 \% \text { males smokers; } \\
1.02 \% \text { female non-smokers }\end{array}$ & $\begin{array}{l}\text { G:1; P: } 5 ; \mathrm{N}: 5 \\
\text { (limited } \\
\text { information re. } \\
\text { objectives, } \\
\text { methods and } \\
\text { analysis) }\end{array}$ \\
\hline
\end{tabular}

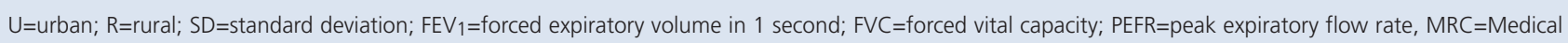
Research Council; $\mathrm{AO}=$ airway obstruction, $\mathrm{NS}=$ non-significant; $\mathrm{S}=$ significant; $\mathrm{G}=$ good; $\mathrm{P}=$ poor; $\mathrm{N}=$ non-assessable'

may not be an estimate of COPD per se, despite the suggested investigation aims. ${ }^{21}$ A second study reporting high prevalence rates used a relatively wide definition of $\mathrm{COPD}^{18}$ and another did not report the spirometric criteria used..$^{27}$ All other studies identified were carried out before 2001. Unusual criteria were used by Malik et al. ${ }^{26}$ and Jindal, ${ }^{23}$ and the remaining two studies ${ }^{22,25}$ had quality concerns. Thus, we found no study from which to draw an estimate of current prevalence using a relatively standard definition. 


\section{Prevalence of chronic bronchitis}

We found prevalences of chronic bronchitis to vary between 3.3\% and $20.9 \%$ in the general population, with all values except this latter figure falling at or below $9.1 \%$. The figures are displayed under 'urban/rural' categorisation in Table 4 and these categories are discussed separately here.

In urban areas, prevalences up to $<9.9 \%$ were reported. However, after exclusion of studies with figures for only urban and rural areas combined or where only a range was provided and those with quality concerns, only two studies remained. These reported prevalences of $1.8 \%{ }^{14}$ and $7.5 \% .^{15}$ The diagnostic criteria for one study were unusually wide, which was possibly the reason for its higher estimate. The $1.8 \%$ estimate is consistent with the others but is reasonably low, particularly as it was derived from an elderly population where relatively high levels would be anticipated, even if only due to the prevalence-incidence bias associated with chronic diseases. Potentially, the 'clinical assessment' included in the study did involve assessment of airway obstruction and the figure quoted relates to COPD, but this is not clear.

Our better estimates of prevalence of chronic bronchitis therefore come from rural populations. Two studies gave a relatively high estimate ${ }^{16,26}$ and another a relatively low estimate. ${ }^{25}$ Suggestions from the authors that these populations were exposed to relatively high levels of domestic pollution and relatively low levels of atmospheric pollution, respectively, may account for this. Estimates from studies without quality concerns were otherwise in relatively good agreement - between $6.5 \%$ and $7.68 \%$ where specific figures were available.

Given the difficulties in interpretation of many of the urban population estimates, we cannot conclude that we have observed different prevalences in rural versus urban regions, although withinstudy comparisons would certainly suggest this may be the case, with higher prevalences in rural areas. ${ }^{13,14,16,18}$

\section{Age}

The prevalences of COPD and chronic bronchitis observed by age category are shown in Table 5. Surprisingly, there was no obvious association with age for either disease. In the case of COPD, the two studies without quality concerns may have been indicative of higher prevalence with advancing age, but the suggestion is confounded by ill-defined diagnostic criteria in one case and unusually wide criteria in the other.

With regard to chronic bronchitis, the lack of association with age is striking. Some association would be anticipated in any chronic disease. Although this is clearly observed within studies, 12,13,16,22,25,26 lack of between-study trends (even when those with quality concerns are excluded) suggest that other variables have a greater impact on prevalence.

Table 4. Prevalence estimates for COPD and chronic bronchitis in rural and urban areas

Estimates are noted as specific 'rural' or 'urban' prevalences where possible, as '<' or '>' a mixed (urban/rural)

estimate where specific estimates have not been made, but a significant difference between residential environments

found, or as an estimate from a mixed urban/rural population where further specifics were unavailable. Estimates

are listed in date order, using date of study where given, or otherwise date of publication (identified by prefix 'P'). Spirometric diagnostic thresholds for diagnosis of COPD are given in parenthesis after the estimates where available

\begin{tabular}{|c|c|c|c|c|c|}
\hline \multirow[t]{2}{*}{ Ref } & \multirow{2}{*}{$\begin{array}{l}\text { Date of } \\
\text { study/ } \\
\text { publication }\end{array}$} & \multicolumn{2}{|l|}{ COPD } & \multicolumn{2}{|c|}{ Chronic bronchitis } \\
\hline & & Rural & Urban & Rural & Urban \\
\hline 21 & P2010 & $58.5 \%(N R)$ & & & \\
\hline 12 & P2010 & & & $7.1 \%$ & \\
\hline 13 & P2006 & & & $>4.1 \%$ & $<4.1 \%$ \\
\hline 14 & $2003-2005$ & & & $7.6 \%$ & $1.8 \%$ \\
\hline 15 & $2002-2003$ & & & & $7.5 \%$ \\
\hline 16 & $2001-2002$ & & & $>9.1 \%$ & $<9.1 \%$ \\
\hline 17 & P2001 & & & & $0.3-5.9 \%$ \\
\hline 18 & $1999-2000$ & $43.1-57.1 \%(N R)$ & $31.4-36.7 \%$ (NR) & & \\
\hline 22 & P1999 & & $5.2 \%\left(F E V_{1} / F V C<0.75\right)$ & & $5.7 \%$ \\
\hline 19 & P1994 & & & $7.68 \%$ & \\
\hline 23 & 1990 & \multicolumn{2}{|c|}{$1.8 \%$ (PEFR < mean minus 1.65 SD of population) } & \multicolumn{2}{|c|}{$3.6 \%$} \\
\hline 24 & P1987 & & & $5.0 \%$ & $2.3-4.5 \%$ \\
\hline 25 & $1981-1986$ & $2.5 \%($ PEFR $<70 \%$ of 'predicted normal') & & $3.3 \%$ & \\
\hline 26 & P1986 & $8.3 \%$ (FEV $1<1.5 L$ (males) or $<1$ L (females)) & & $20.9 \%$ & \\
\hline 20 & P1982 & & & $6.5 \%$ & \\
\hline 27 & 1981 & & $0.6-4.27 \%(N R)$ & & $0.89-9.9 \%$ \\
\hline
\end{tabular}

$\mathrm{NR}=$ not reported; $F E V_{1}=$ forced expiratory volume in 1 second; FVC=forced vital capacity; PEFR=peak expiratory flow rate; SD=standard deviation. Studies of quality concern (see Methods) are entered in italics. 


\section{Sex}

Within-study testing was again suggestive that both COPD and chronic bronchitis are more frequent in males than females. This was not evident between studies for COPD, but, in studies of chronic bronchitis, males did have generally higher estimated rates of disease once those with quality concerns were excluded.

\section{Smoking and domestic smoke exposure}

The effect of smoking was considered in 11 studies and the effect of domestic smoke exposure/poor ventilation in six. Both were positively associated with disease in all cases. The absolute figures are difficult to interpret when there are considerably higher rates of smoking in males, higher rates of domestic smoke exposure in females, and putative synergistic effect of domestic smoke exposure and smoking. Nevertheless, the effect of smoking does seem to be of considerable magnitude regardless of study setting. The effect of domestic smoke exposure seems to be more sensitive to the sampling frame.

\section{Discussion}

\section{Main findings}

We planned an investigation to determine the prevalence of COPD in India, any time trends in prevalence, and effect of subgroup. A key finding was the paucity of data and inconsistency in study setting and population characteristics. Figure 2 shows the 10/28 Indian states in which studies have been performed. Study quality was also variable and of concern in 7/16 cases, although more improved in recent years. Because of these issues, we were unable to give a useful estimate of COPD prevalence or to draw conclusions regarding time trends of COPD prevalence. Assessment of subgroup effect was severely limited. We were not able to give any prevalence estimates with measures of confidence.

It was disappointing that we did not identify any study that could provide a rigorous estimate of COPD prevalence using a relatively standard definition. We were better able to review prevalences of chronic bronchitis, particularly in rural populations. This was most consistently reported as between $6.5 \%$ and $7.68 \%$, but higher and lower figures were also reported and potentially associated with peculiarities in the environments of the studied populations. That particular subpopulations may have quite different prevalences has been shown in relatively controlled study. ${ }^{13}$

Although several of the reviewed studies themselves demonstrated higher prevalences in rural versus urban regions, lack of suitable urban studies hindered any further analysis in this regard. Similarly, we could not convincingly demonstrate an effect of age or domestic smoke exposure further than that suggested by individual studies. Only sex and smoking status seemed to show an effect on the prevalence of chronic bronchitis great enough to be evident despite the heterogeneity between studies, potentially suggesting that they are of relatively great effect. As nearly all smokers in the reviewed studies were male, it is unclear to what extent the effect of these variables may be mediated through each other.

\section{Table 5. Prevalence estimates for COPD and chronic bronchitis by age range}

Prevalence estimates listed by age range of samples. Age ranges of the particular studies start between the ranges given below. Only study 24 had an upper limit (of 80 years)

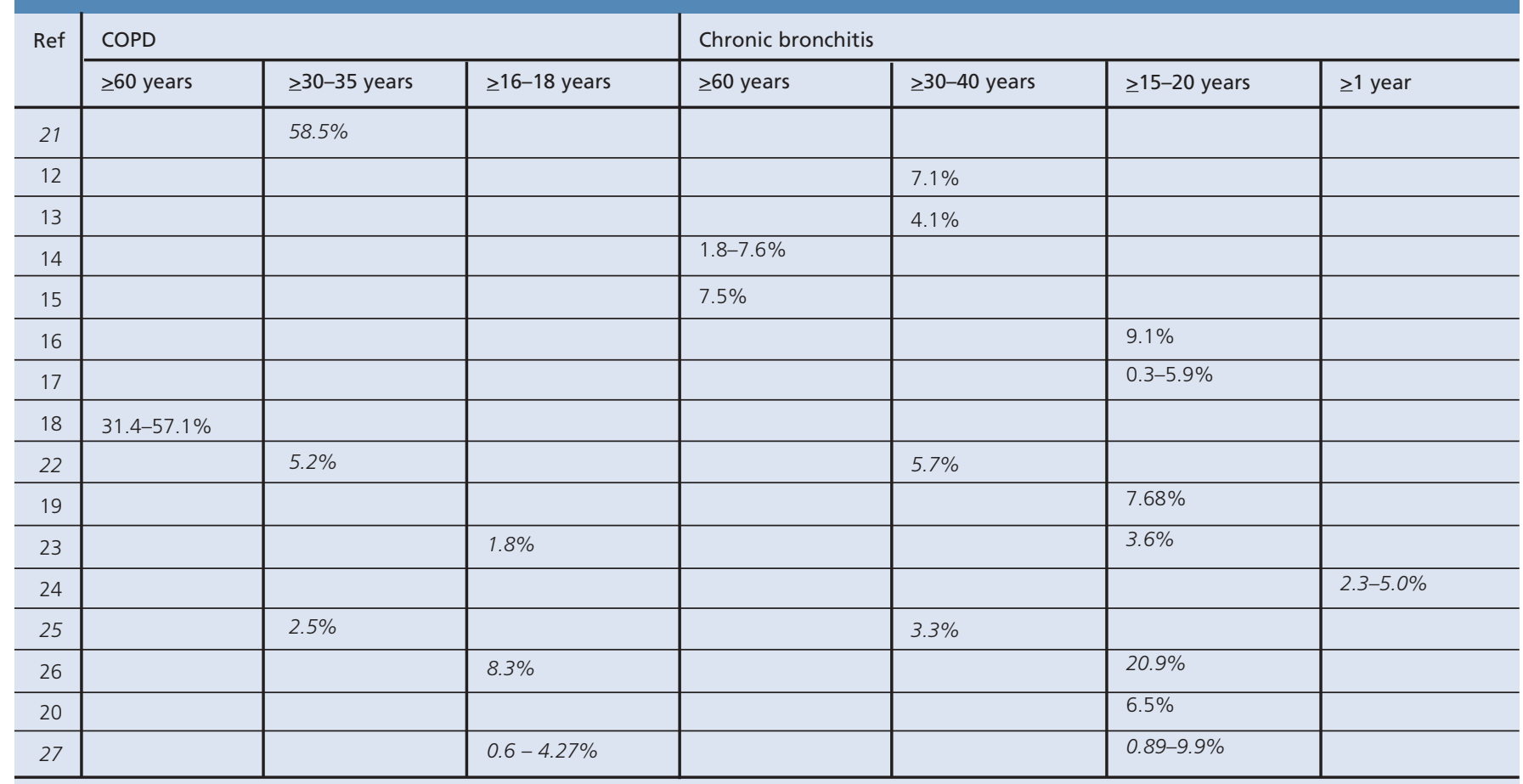

$\mathrm{NR}=$ not reported; $F E V_{1}=$ forced expiratory volume in 1 second; $F V C=$ forced vital capacity; $P E F R=$ peak expiratory flow rate; $S D=s t a n d a r d ~ d e v i a t i o n$. Studies of quality concern (see Methods) are entered in italics. 


\section{Interpretation of findings in relation to previously published work}

Although a systematic review of COPD prevalence in India has not, to our knowledge, been carried out previously, Jindal reviewed some of the literature in $2006^{\circ}$ and Murthy and Sastry in 2005. ${ }^{8}$ Neither of these studies involved any synthesis but, in an earlier review, Jindal suggested a median COPD prevalence of $5 \%$ in males and $2.7 \%$ in females..$^{30}$ In fact, these estimates are probably solely for chronic bronchitis and thus are below those we have presented. This may be because our estimate was drawn from entirely different studies: almost all of the newer studies they reviewed are included in our study but none were included in the analysis due to quality concerns.

Comparisons with other countries are difficult as estimates from many neighbouring countries as well as India are lacking. However, in 2003 a Regional COPD Working Group used prevalences of various risk factors to estimate the prevalence of COPD in the whole Asia-Pacific region. ${ }^{31}$ Their estimate was $6.3 \%$, in keeping with our observations here. This study was also indicative that COPD rates in India are similar - or perhaps slightly higher - than those in Europe and North America, but potentially lower than in some neighbouring countries. In contrast, data from the Burden of Obstructive Lung Disease (BOLD) study indicate that the prevalence in more developed countries may be slightly higher than those in India. ${ }^{32}$

Of course the burden in India is nevertheless relatively great ${ }^{6}$ and may be expected to increase as the population ages. However, the Indian population is currently relatively male and there are relatively high levels of use of indoor fuels and inadequate ventilation. Political, social, and economic climates will be important, but hopefully advances in these areas can help stem any growth in COPD prevalence. Popularity of smoking will also be important. Only as trends in these variables become easier to predict will trends in COPD become similarly so.

\section{Strengths and limitations of this study}

Although the low number and heterogeneity of the reviewed studies were a notable outcome in themselves, they did have a considerable effect on the extent to which this review could be undertaken as planned. Further limitations on our results were imposed by (1) aspects of the review methodology and (2) further issues concerning the availability of supplementary information. The methodology was constrained in particular by the subjective aspect of quality assessment and use of quality ratings, and the lack of appropriateness of data for meta-analysis. With regard to availability of supplementary information, it seems unlikely that we have had access to all available data as the BOLD database is suggestive that additional prevalence studies in India - not obviously widely available - have been completed..$^{33}$

However, it is likely that through our thorough and systematic approach, we have identified most of the available data and, although the data were not suitable for meta-analysis, the systematic approach is a useful and clear method for providing a data summary and clearly demonstrating where gaps exist.

\section{Implications for future research, policy and practice}

In order to obtain a better understanding of the prevalence of COPD in India, it would be useful to use prospective, ongoing, standardised data
Figure 2. States in which studies have been conducted. States wherein prevalence of chronic obstructive pulmonary disease/chronic bronchitis has been estimated for subpopulations are indicated with black dots

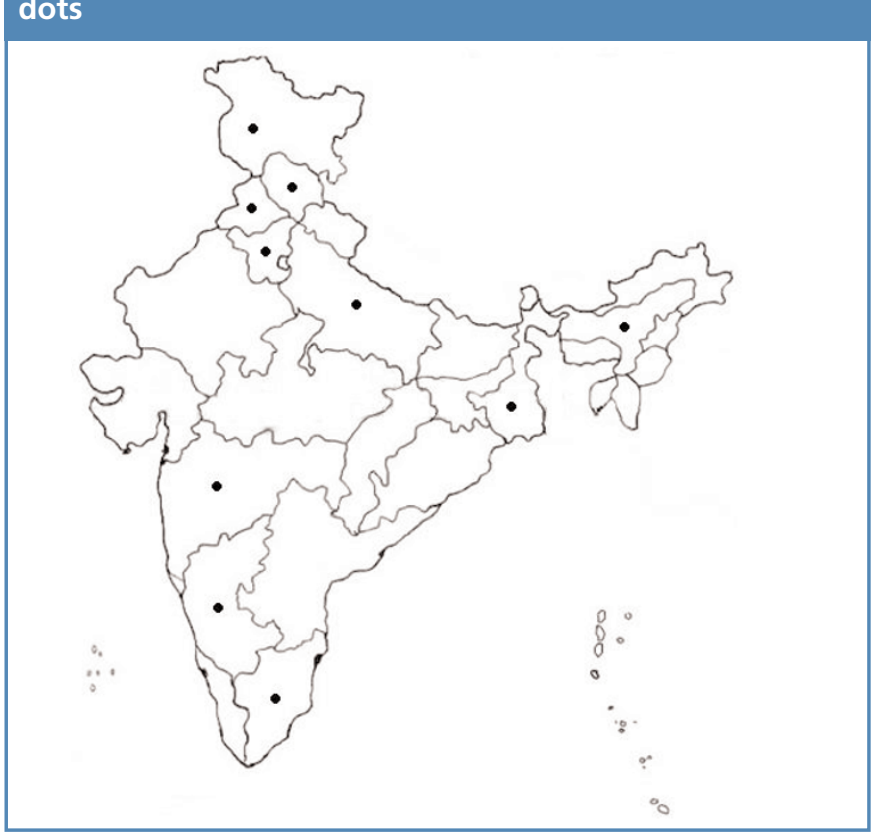

collection - preferably from samples of the entire population, including those that do not typically access healthcare - to assess prevalence and time trends, including by subgroup. Such basic information would provide a useful background on which study of more subtle themes such as those relating to the nature of disease amongst subgroups and how best to target resources - could be based.

The difficulty in sampling the 'entire population' of such a diverse country is acknowledged, particularly when the distribution of important variables such as smoking, domestic smoke exposure, and outdoor pollution is ill-defined. A useful starting point would be to define the various major 'types' of population in the country (based, for example, on natural environment, socioeconomic status and ethnicity), and to begin ongoing, rigorous, standardised measurements of prevalence in a series of areas which, when combined, could give an approximation of the general country prevalence. Moreover, this would help demonstrate different prevalences/trends between the types of region and thus where different management approaches might be useful. Implementation of data collection as an ongoing part of clinical practice would be optimal but perhaps still impractical, at least in some areas where repeated cross-sectional studies may be required meanwhile. General population cross-sectional studies would also have the advantage of detecting cases that would not otherwise present to healthcare services.

\section{Conclusions}

The current prevalence of COPD in India is unclear. Accurate estimates of current/future disease prevalence are required for optimal strategies to mitigate growth and effects of disease to be devised. Thorough standardised prospective ongoing epidemiological 
study of the many and various Indian subpopulations will be necessary. Understanding current prevalences of risk factors and their likely trends will also be helpful. Once a background of rigorous general prevalence estimations is in place, there will be opportunity to investigate the variations associated with particular subpopulations.

\section{Handling editor Sundeep Salvi \\ Statistical review Gopal Netuveli}

Conflicts of interest The authors declare they have no conflicts of interest in relation to this article.

Contributorship AJM, MPA and AM contributed to study design. Data were collected by AJM and JZF. Analysis was carried out by AJM. AJM, MPA and AM drafted/revised the article. The final version has been reviewed and approved by all authors.

Funding This review forms part of a project for which funding was awarded to AJM by the Association of Commonwealth Universities and the Royal College of Physicians of London. The funders played no role in study design, data collection or interpretation, the writing or dissemination of this review. The Department of Primary Care \& Public Health at Imperial College London is grateful for support from the NIHR Collaboration for Leadership in Applied Health Research \& Care (CLAHRC) Scheme and the NIHR Biomedical Research Centre scheme.

\section{References}

1. Report of the Secretary General. Prevention and control of non-communicable diseases. Item 119; United Nations 66th session, 2011. Available from: http://www.un.org/ga/search/view_doc.asp?symbol=A/66/83\&Lang=E (accessed Dec 2011).

2. World Health Organization. Global status report on non-communicable diseases 2010. World Health Organization, 2011. Available from:

http://www.who.int/nmh/publications/ncd_report_full_en.pdf (accessed Dec 2011

3. Global Risks 2010: A Global Risk Network Report, World Economic Forum, January 2010. World Economic Forum, 2010

4. World Health Organization. Chronic obstructive pulmonary disease (COPD) Fact sheet No 315. World Health Organization, 2011. Available from: http://www.who.int/mediacentre/factsheets/fs315/en/index.html (accessed July 2011).

5. Department of Measurement and Health Information Systems of the Information, Evidence and Research Cluster. World Health Statistics 2008. World Health Organization, 2008. Available from:

http://www.who.int/whosis/whostat/EN_WHS08_Full.pdf (accessed Aug 2011).

6. Lopez AD, Mathers CD, Ezzati M, Jamison DT, Murray CJL. Global burden of disease and risk factors. The International Bank for Reconstruction and Development/The World Bank Group, 2006.

7. Halbert RJ, Isonaka S, George D, Iqbal A. Interpreting COPD prevalence estimates. What is the true burden of disease? Chest 2003;123:1684-92. http://dx.doi.org/10.1378/chest.123.5.1684

8. Murthy KJR, Sastry JG. Economic burden of chronic obstructive pulmonary disease. National Commission on Macroeconomics and Health Background Papers - Burden of Disease in India. National Commission on Macroeconomics and Health, Government of India, 2005. Available from: http://www.who.int/macrohealth/ action/NCMH_Burden\%20of\%20disease_\%2829\%20Sep\%202005\%29.pdf (accessed Sep 2011).

9. Jindal SK. Emergence of chronic obstructive pulmonary disease as an epidemic in India. Indian J Med Res 2006;124:619-30.

10. Moher D, Liberati A, Tetzlaff J, Altman DG, The PRISMA Group. Preferred reporting items for systematic reviews and meta-analyses: the PRISMA statement. PLoS Med 2009;6:e1000097. http://dx.doi.org/10.1371/journal.pmed.1000097

11. Centre for Reviews and Dissemination. Systematic reviews: CRD's guidance for undertaking reviews in health care. Centre for Reviews and Dissemination, University of York, 2008. Available from:

http://www.york.ac.uk/inst/crd/pdf/Systematic_Reviews.pdf (accessed April 2011).
12. Mahesh PA, Jayaraj BS, Prahlad ST, et al. Validation of a structured questionnaire for COPD and prevalence of COPD in rural area of Mysore: a pilot study. Lung India 2009;26:63-9. http://dx.doi.org/10.4103/0970-2113.53226

13. Jindal SK, Aggarwal AN, Chaudhry K, et al; Asthma Epidemiology Study Group. A multicentric study on epidemiology of chronic obstructive pulmonary disease and its relationship with tobacco smoking and environmental tobacco smoke exposure. Indian J Chest Dis Allied Sci 2006;48:23-9.

14. Sousa RM, Ferri CP, Acosta D, et al. Contribution of chronic diseases to disability in elderly people in countries with low and middle incomes: a 10/66 Dementia Research Group population-based survey. Lancet 2009;374:1821-30. http://dx.doi.org/10.1016/S0140-6736(09)61829-8

15. Medhi GK, Hazarika NC, Borah PK, Mahanta J. Health problems and disability of elderly individuals in two population groups from same geographical location. J Assoc Physicians India 2006;54:539-44.

16. Goel S, Gupta BP, Kashyap S, Bhardwaj AK. Epidemiological aspects of chronic bronchitis in Shimla hills. Indian J Chest Dis Allied Sci 2007;49:144-7.

17. Chhabra SK, Chhabra P, Rajpal S, Gupta RK. Ambient air pollution and chronic respiratory morbidity in Delhi. Arch Environ Health 2001;56:58-64. http://dx.doi.org/10.1080/00039890109604055

18. Joshi K, Kumar R, Avasthi A. Morbidity profile and its relationship with disability and psychological distress among elderly people in Northern India. Int J Epidemiol 2003;32:978-87. http://dx.doi.org/10.1093/ije/dyg204

19. Qureshi KA. Domestic smoke pollution and prevalence of chronic bronchitis/asthma in a rural area of Kashmir. Indian J Chest Dis Allied Sci 1994;36:61-72.

20. Nigam P, Verma BL, Srivastava RN. Chronic bronchitis in an Indian rural community. J Assoc Physicians India 1982;30:277-80.

21. Mukherjee R, Moore VC, Purkait S, et al. Feasibility of performing valid spirometry in rural India: preliminary results from a population study assessing the prevalence of COPD. Conference Publication: British Thoracic Society Winter Meeting 2010, London, UK. Thorax 2010;65:A129. http://dx.doi.org/10.1136/thx.2010.150987.22

22. Akhtar MA, Latif PA. Prevalence of chronic bronchitis in urban population of Kashmir. J Indian Med Assoc 1999;97:365-9.

23. Jindal SK. A field study on follow up at 10 years of prevalence of chronic obstructive pulmonary disease and peak expiratory flow rate. Indian J Med Res 1993;98:20-6.

24. Kamat SR, Doshi VB. Sequential health effect study in relation to air pollution in Bombay, India. Eur J Epidemiol 1987;3:265-77. http://dx.doi.org/10.1007/BF00149735

25. Ray $D$, Abel R, Selvaraj KG. A 5-yr prospective epidemiological study of chronic obstructive pulmonary disease in rural south India. Indian J Med Res 1995;101:23844.

26. Malik SK, Kashyap S. Chronic bronchitis in rural hills of Himachal Pradesh, northern India. Indian J Chest Dis Allied Sci 1986;28:70-5.

27. Malik SK, Banga N, Qamra S. Chronic bronchitis in Chandigarh, North India. Bulletin Postgraduate Institute of Medical Education and Research, Chandigarh 1981;15:161-3.

28. American Thoracic Society. Standards for the diagnosis and care of patients with chronic obstructive pulmonary disease (COPD) and asthma. Am Rev Respir Dis1987;136: 225-44. http://dx.doi.org/10.1164/ajrccm/136.1.225

29. Global Initiative for Chronic Obstructive Lung Disease (GOLD). Global Strategy for the Diagnosis, Management and Prevention of COPD. Global Initiative for Chronic Obstructive Lung Disease, 2011. Available from: http://www.goldcopd.org/ (accessed Feb 2012).

30. Jindal SK, Aggarwal AN, Gupta D. A review of population studies from India to estimate national burden of chronic obstructive pulmonary disease and its association with smoking. Indian J Chest Dis Allied Sci 2001;43:139-47.

31. Regional COPD Working Group. COPD prevalence in 12 Asia-Pacific countries and regions: projections based on the COPD prevalence estimation model. Respirology 2003;8:192-8. http://dx.doi.org/10.1046/j.1440-1843.2003.00460.x

32. Buist AS, McBurnie MA,Vollmer WM, et al., on behalf of the BOLD Collaborative Research Group. International variation in the prevalence of COPD (The BOLD Study): a population-based prevalence study. Lancet 2007;370:741-50. http://dx.doi.org/10.1016/S0140-6736(07)61377-4

33. BOLD Study - Burden of Obstructive Lung Disease: Sites. Available from: http://www.boldstudy.org/sites.html (accessed Sep 2011). 


\section{Appendix. PRISMA 2009 Checklist}

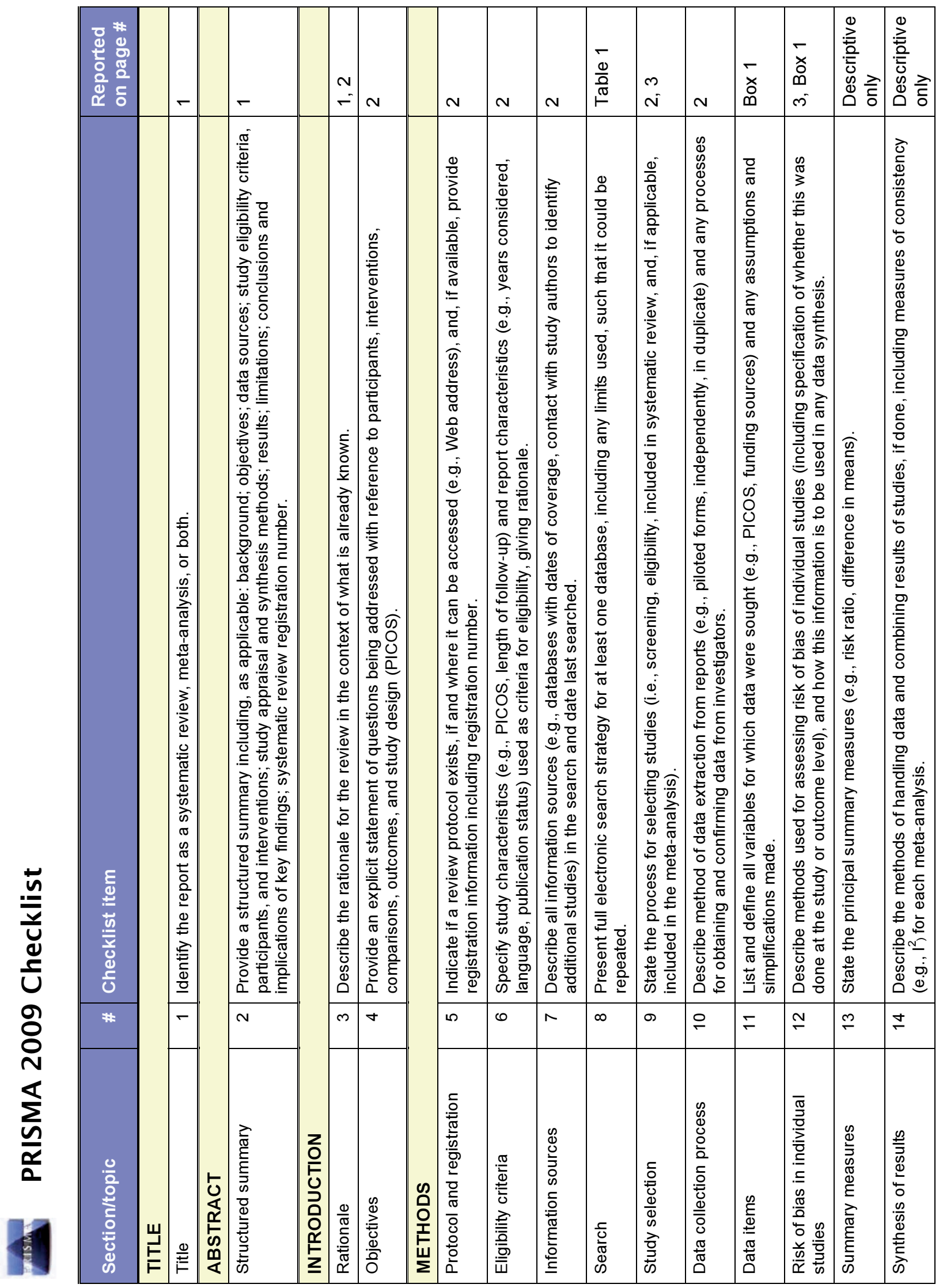




\section{Appendix. PRISMA 2009 Checklist continued}

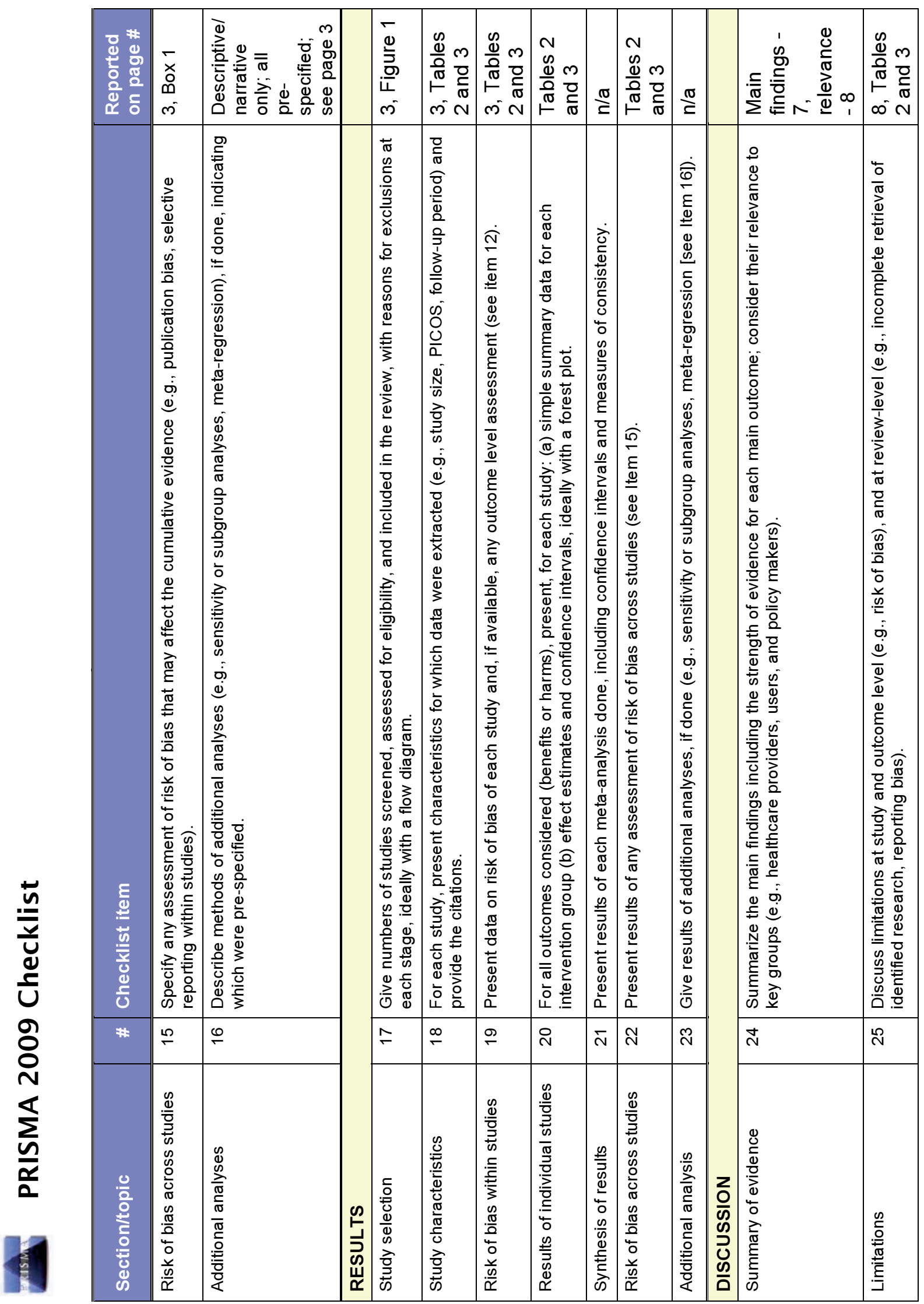


Appendix. PRISMA 2009 Checklist continued

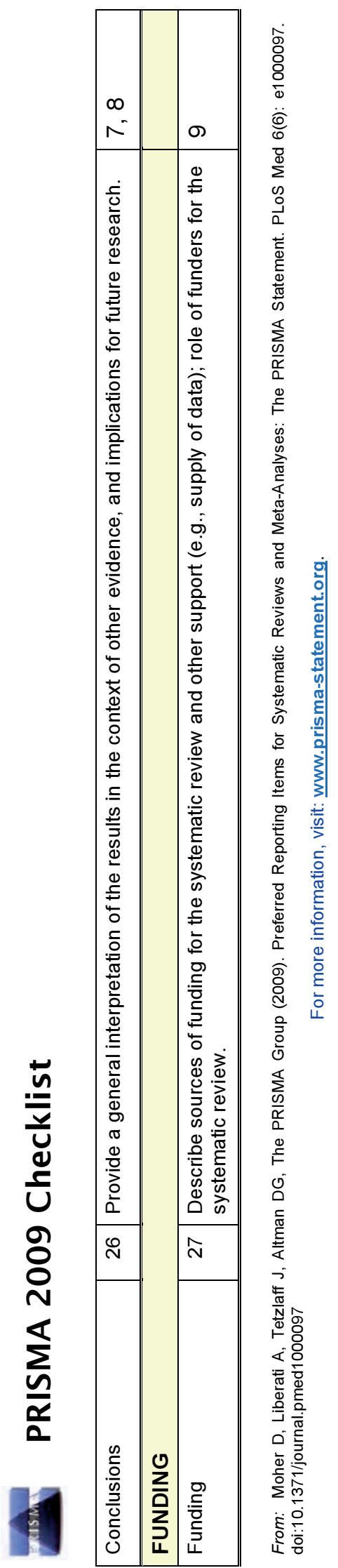

PRIMARY CARE RESPIRATORY JOURNAL 